\title{
Análisis de la situación del consumo de pollo en tiendas de barrio
}

\section{Analysis of the situation of chicken consumption in neighborhood stores}

\author{
Mailin Dayana Verjel - Carrascal ${ }^{1}$, Carlos Alberto Pacheco - Sánchez ${ }^{2}$ \\ ${ }^{1}$ Estudiante, Programa de Administración de Empresas, Universidad Francisco de Paula Santander Ocaña, Colombia \\ ORCID: : https://orcid.org/0000-0001-8670-8023,E-mail: mdverjelc@ufpso.edu.co \\ ${ }_{2}^{2}$ Magister, Administrador de Empresas, Universidad Francisco de Paula Santander Ocaña, Colombia, \\ ORCID: https://orcid.org/0000-0002-9369-582X, E-mail: capachecos@ufpso.edu.co
}

Cómo citar: Verjel - Carrascal, D. M., \& Pacheco - Sánchez, C. A. (2018). Análisis de la situación del consumo de pollo en tiendas de barrio. Revista Científica Profundidad Construyendo Futuro, 9(9), 10-15. https://doi.org/10.22463/24221783.2609

Recibido: 15 de Enero de 2018 / Aprobado: 22 de Marzo de 2018

\begin{abstract}
Resumen
En la canasta familiar, la carne de pollo se ha convertido en uno de los principales productos para las familias del país, por tanto, la investigación busco conocer la dinámica de la oferta del consumo de pollo a través de la metodología descriptiva con enfoque cuantitativo, para ello, se trabajó con una población de 28 tiendas de barrio ubicadas en la comuna 4 de Ocaña de acuerdo a los datos obtenidos por Cámara de Comercio de Ocaña. Dentro de los resultados obtenidos se comprende que la comunidad adquiere productos por unidad o peso, asimismo, se logra observar que más del 20\% de los tenderos logran comercializar de 1 a 2 pollos durante el día.
\end{abstract}

Palabras claves: Consumo, Carne de pollo, Tiendas de barrio, ventas.

\begin{abstract}
In the family basket, chicken meat has become one of the main products for families in the country, therefore, the research sought to know the dynamics of the supply of chicken consumption through the descriptive methodology with a quantitative approach, for this, we worked with a population of 28 neighborhood stores located in Ocaña commune 4 according to data obtained by the Ocaña Chamber of Commerce. Among the results obtained, it is understood that the community purchases products per unit or weight, and it is also observed that more than $20 \%$ of the storekeepers manage to market 1 to 2 chickens during the day.
\end{abstract}

Key words: Consumption, Chicken meat, Neighborhood shops, sales. 


\section{Introducción}

En Ocaña, carecer de datos o estadísticas que permitan evaluar el consumo de pollo y demás bienes de la canasta familiar en las diferentes comunas es un problema para evaluar la economía, por tanto, con el estudio se podría conocer las condiciones y el comportamiento de compra de pollo, con lo anterior se sustenta que el bien es un producto consumido por las familias colombianas, ya que es un acompañamiento a las raciones diarias. Partiendo de ello, surge el interrogante ¿Qué tanto se consume y se vende la carne de pollo?

Y es que la producción de carne de pollo y huevo alcanzó un nuevo récord el año pasado. La primera llegó a 1,47 millones de toneladas, mientras la segunda a los 12.800 millones de unidades. Esta producción conjunta vale alrededor de $\$ 16,5$ billones, dice Andrés Valencia, presidente de la Federación Nacional de Avicultores. Estas cifras ponen en evidencia un incremento en el consumo per cápita de estas dos proteínas. El año pasadocada colombiano consumió en promedio 31,5 kilos de carne de pollo y la proyecciónes que para 2017 esté alrededor de 32 kilos. En huevos el dato llegó a 272 unidades por persona y para este año podríaubicarse entre $275 \mathrm{y}$ 280. (Dinero, 2017)

Tras el crecimiento de la producción de pollo se puede asimilar que cada vez más se logra percibir el gran desarrollo avícola que aporta a la economía, pero algo que lleva a que este producto sea tan consumido es porque su precio se ha mantenido a pesar de ser tan recurrido por sus consumidores.

En el caso del precio, hay que decir que, a diferencia de otras proteínas animales, el pollo y huevo no registran incrementos tan altos en los últimos años. Según estadísticas de FENAVI, el kilo de pollo se mantiene desde hace años entre los $\$ 5.800$ y $\$ 6.500$. "Los productores y granjas se han dedicado a ser más productivos y competitivos y eso ha llevado a que los precios se mantengan", comenta Gonzalo Moreno, presidente de FENAVI (Dinero, 2019).

Es un respiro a los hogares colombianos ya que el producto solo ha tenido un incrementoen el precio de venta promedio de $\$ 700$, por lo anterior, es un producto sustituyente donde los consumidores seguirán adquiriendo su misma cantidad ya que ven el pollo más saludable y barato que otro tipo de carnes.

Pero muchas personas no conocen cuales son los beneficios y propiedades de consumirdicho alimento:

Las propiedades nutricionales, la competitividad del precio y la producción saludable son algunas de las razones del crecimiento del consumo de este alimento. Máspollo, menos carne. Esa es la tendencia en el menú de los colombianos, de acuerdo con un informe de la universidad de La Salle en el que se explican las razones del crecimiento del consumo de la producción avícola frente a la caída de la demanda de la carne roja en los últimos 10 años, especialmente desde 2007, cuando el DANE, informó que el consumo de este tipo era de 18 kilogramos por persona al año y, en contraste, el del pollo era de 19 kilogramos. (EL ESPECTADOR, 2012)

Asimismo, EL SIGLO (2018) afirma que "El pollo es la carne favorita de los nutriólogos. No es raro escuchar que sea la primera que está incluida en las dietas de sus pacientes. Esto se debe a que consumirla aporta múltiples beneficios al organismo". Esto ayuda al mantenimiento de nuestro peso, a tener una salud adecuada, son algunas de las 
razones del por qué se debe consumir este alimento.

Con base a lo anterior según datos de FENAVI hay aún más razones por las cuales este alimento es uno de los más beneficiarios y de los cuales se debe consumir, abarcando antes que el pollo no pasa por ningún tipo de hormonas para su crecimiento y formación, por esto mencionan las 6 razones para comer pollo. "Es alto en proteína, la grasa contenida en el pollo es saludable, es rico en vitaminas, es rico en fósforo, antidepresivo natural, es un alimento con gran versatilidad gastronómica" (Angarita, 2017). Según dice la revista, algunas de las partes del pollo aportan hasta el $50 \%$ de los nutrientes necesarios para el cuerpo; las grasas no afectan tanto al consumidor, tiene el fosforo que es un mineral esencial que ayuda a los dientes y huesos; obtiene triptófano que es un amoniaco en el cual cumple la saciedad del usuario y tiene múltiples preparaciones para cumplir el paladar de la persona. Finalmente, el objetivo que se cumple mediante la investigación es analizar la oferta de consumo de pollo en las tiendas de barrios de la Comuna 4 del municipio de Ocaña, aplicando la muestra presentada por la Cámara de Comercio.

\section{Marco Teórico}

Para la investigación se apoya a las siguientes teorías:

\subsection{Teoría de la segmentación del mercado}

La teoría de la segmentación de mercados ayuda a la toma de decisiones para el marketing.

El proceso de segmentación de mercados constituye en enfoque sistemático para guiar el proceso de toma de decisiones en marketing. Este proceso consiste en dividirun mercado heterogéneo en partes. Los segmentos identificados han de ser homogéneos en su interior, sí, pero heterogéneos en relación con el exterior esto es, respecto a otros segmentos). La finalidad es facilitar el diseño de programasespecíficos de marketing que sean los másadecuados para estos segmentos particulares. Esto es posible porque los estudios de segmentación revelan las características y necesidades de los usuarios potenciales de productos y servicios. (Kotler, 1991,p.276)

La segmentación de mercados se enfoca en identificar y determinar grupos con características homogéneas, en los cuales la organización pueda dirigirse para obtener resultados.

\subsection{Teoría del consumidor}

La teoría del consumidor da a conocer como es el carácter de la persona al consumirbien o servicios para satisfacer sus necesidades.

Cabe destacar, quizá como excepción a este olvido del ser de la teoría neoclásica, el aporte que Kevin Lancaster realiza a la teoría del consumidor, cuando establece que lo importante no son los bienes en sí mismos sino sus características, o en otras palabras los servicios que los bienes brindan a los consumidores. (Fernández, 2009)

La teoría del consumidor, analiza como es el comportamiento del mismo al momento deinteractuar con un producto o servicio y su actividad presupuestaria para obtenerla. 


\section{Metodología}

La investigación cuantitativa de tipo descriptivo, realiza una descripción de la oferta de carne de pollo, por otra parte, se aplicó la técnica de la encuesta a las tiendas ubicadas en los barrios de la comuna $\mathrm{N}^{\circ} 4$ del municipio de Ocaña.

Tabla 1. Barrios de la comuna 4 registrados en lacámara de comercio

\begin{tabular}{lc}
\hline \multicolumn{2}{c}{ UBICACIÓN } \\
\multicolumn{1}{c}{ BARIO } & CANTIDAD \\
\hline 00099-TORCOROMA & 1 \\
00100-MARABELITO & 4 \\
00101-MARABEL & 2 \\
00102-CUARTO & 5 \\
CENTENARIO & 5 \\
100105-PRIMERO DE & 2 \\
MAYO & 3 \\
00108-TEJARITO & \\
00110-CAMILO & 3 \\
TORRES & 1 \\
00114-LANDIA & \\
00116-NUEVA & 6 \\
ESPAÑA & $\mathbf{2 8}$ \\
00144-JUAN XXIII & \\
TOTAL GENERAL & \\
\hline
\end{tabular}

Nota: En esta tabla se observan 10 barrios de la comuna 4 las cuales están registradas ante (CÁMARA DE COMERCIO, 2019)

Con lo descrito, la muestra estuvo conformada por los tenderos que se encuentran registrados en cámara de comercio, con disposición para dar información y con más de un (1) año de funcionamiento.

\section{Resultados y Discusiones}

Al momento de analizar la oferta de carne de pollo en las tiendas de barrio la Comuna 4 sur occidental Adolfo Milanés, la cual cuenta con 15 barrios (PLAN DE DESARROLLO DEL MUNICIPIO DE OCAÑA 2008-2011), se obtuvieron los siguientes resultados:

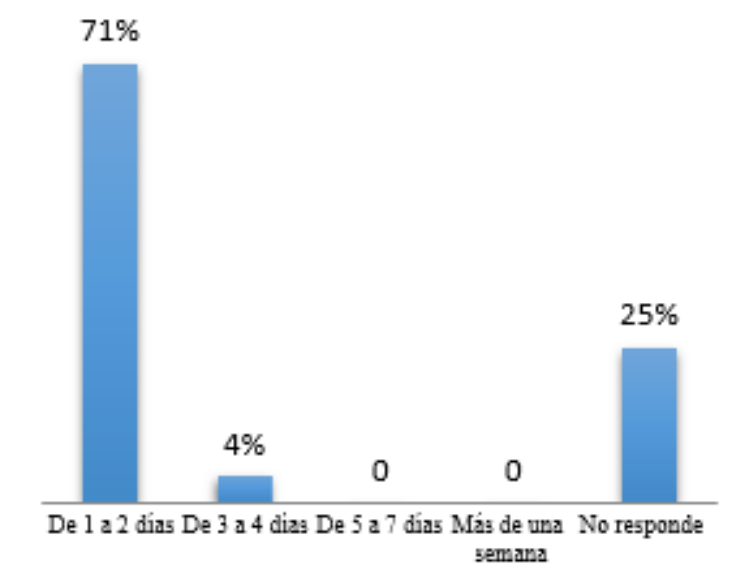

Figura 1. Tiempo de venta de la carne de pollo. Fuente: Elaboración propia.

El proceso de venta de pollo en las tiendas de barrio se realiza de manera diaria, donde el $71 \%$ responden que, entre uno y dos días, tarda el proceso de vender la totalidad del mismo. Por otra parte, solo el $4 \%$ de los tenderos encuestados dio la respuesta de 3 a 4 días informando que lo mantiene en refrigeración hasta venderlo todo.

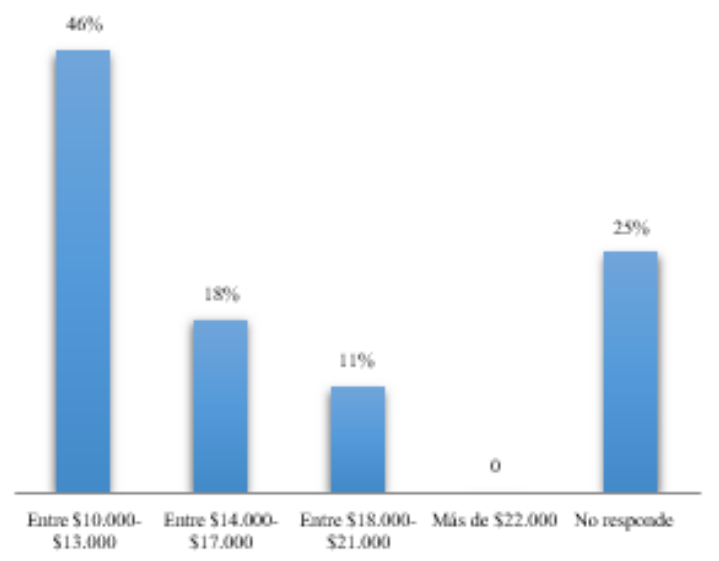

Figura 2. Precio de compra por unidad. Fuente: Elaboración propia. 
La disposición de pago de los tenderos frentea la compra del producto en su local dio a conocer que depende del peso que obtenga enkilogramos, sin embargo, el $46 \%$ de los tenderos afirma que prefieren destinan un ingreso para pago en un rango de $\$ 10.000 \mathrm{y}$ $\$ 13.000$, el $18 \%$ de los tenderos ente $\$ 14.000$ y $\$ 17.000$ y el $11 \%$ cancelan un promedio de $\$ 18.000$ a $\$ 21.000$.

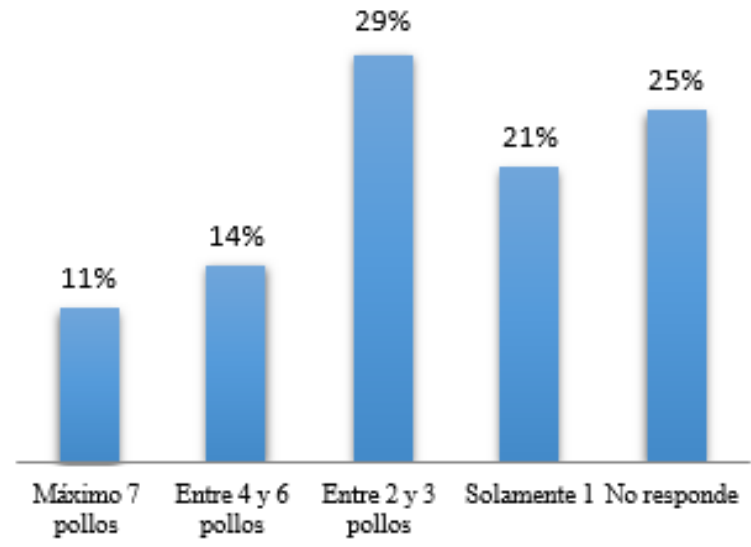

Figura 3. Demanda de pollo por día. Fuente:Elaboración propia.

Con relación a la demanda de pollo, se observa que, en las tiendas de barrio, el $11 \%$ de los tenderos venden máximo 7 pollos, el $14 \%$ entre 4 y 6 pollos, el $21 \%$ esta son solamente un solo pollo en la venta de su tienda, el $29 \%$ entre 2 y 3 pollos. Finalmente, el $25 \%$ no responde, al no tener un control claro del mismo.

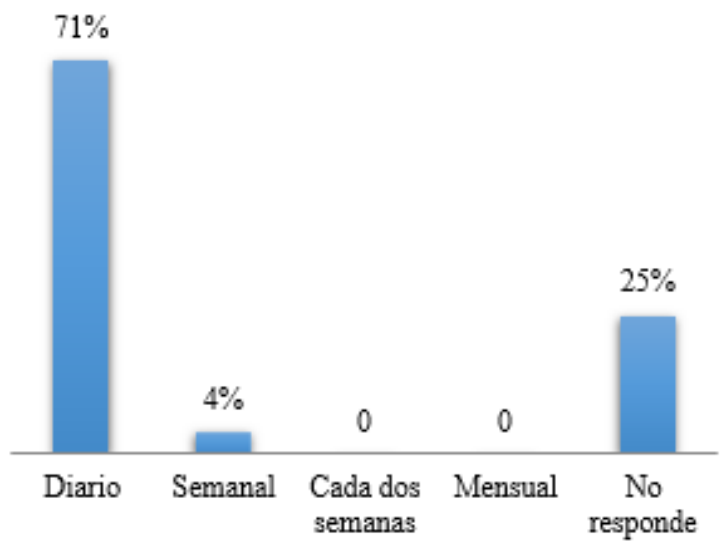

Figura 4. Frecuencia de compra por parte de losclientes. Fuente: Elaboración propia.

Partiendo de los hallazgos, se obtuvo que el $71 \%$ de los clientes van a sus tiendas todos los días a comprar el pollo, solo el $4 \%$ anunció que lo hacen de manera semana (fines de semana), con lo descrito, los clientes acuden diariamente a comprar el pollo, es decir, se sustenta que la población realiza un proceso diario debido a que los ingresos son menos de 1 SMMLV, no tienen salario fijo y la ocupación de los jefes de hogar es de tipo independiente.

\section{Conclusiones}

Con la ejecución de proyecto se puede dara conocer que el consumo de pollo en una delas comunas de Ocaña es constante puesto que en las tiendas hay un porcentaje alto de compra de pollo y se vende diariamente. Por otra, se obtiene que 4 de cada 5 tiendas realizacompra diaria de pollo y se comercializa en un tiempo inferior a 3 días.

Por otra parte, se deduce que el rango promedio de precio de compra de carne de pollo, oscila en un rango de $\$ 12.000$ a $\$ 19.000$ para año 2018. Desde luego, los tenderos se preocupan por adquirir carne 
fresa y de alta calidad debido a las exigencias de los consumidores.

Finalmente, la compra y venta de pollo enOcaña es constante y diaria; en su efecto, se debe a los ingresos que reciben los clientes, asimismo, existe desde la realidad comercial y en consideración de la calidad de vida del cliente, la oferta de despiece de pollo para lograr satisfacer sus necesidades.

\section{Referencias}

Angarita, C. (9 de Noviembre de 2017). Seis razones para comer pollo. Obtenido de https://avicultura.info/seisrazones-comer-pollo/

CAMARA DE COMERCIO. (2019). Estadísticas.

Dinero. (16 de Marzo de 2017). Por qué la industria avícola colombiana está volando alto. Obtenido de https://www.dinero.com/edicionimpresa/negocios/articulo/como-vala-industria-avicola-encolombia/242959

Dinero. (21 de Enero de 2019). El pollo y el huevo van ganando la guerra de la proteína. Obtenido de https://www.dinero.com/pais/articulo /aumenta-la-produccion-y-consumo- depollo-y-huevo/266218

EL ESPECTADOR. (15 de Agosto de 2012). Colombianos comen más pollo que carne. Obtenido de https://www.elespectador.com/notici as/actualidad/colombianos-comenmas-pollo-carne-articulo-367799
EL SIGLO. (11 de Julio de 2018). Razones para comer pollo. Obtenido de htps://www.elsiglodedurango.com. $\mathrm{m} \quad \mathrm{x} /$ noticia/976898.razones-paracomer-pollo.html

FENAVI. (2019). Estadísticas. Obtenido de https://www.redalyc.org/pdf/3579/3 57935473003.pdf

Fernández, R. (2009). Teoría del consumidor. Obtenido de https://www.redalyc.org/pdf/3579/3 5 7935473003.pdf

Kotler. (1991). Segementación de mercados Obtenido de https://www.mapa.gob.es/ministerio /ags/biblioteca/fondo/pdf/5269_39.p df

PLAN DE DESARROLLO DEL MUNICIPIODE OCAÑA 20082011. (s.f.). Obtenido de https://ocananortedesantander.mico lombiadigital.gov.co/sites/ocananort edesantander/content/files/000069/3 443 _acuerdo_pd_oca_a_20.pdf 